\title{
Research methods in human cardiovascular pharmacology
}

Cardiovascular disease is the major cause of morbidity and mortality in the developed world. This position is likely to remain unchallenged as these populations live longer, become increasingly affluent and adopt a more sedentary lifestyle. For the same reasons, cardiovascular disease is now emerging as a significant problem in the populations of the developing world. During the 20th century, as the extent of the problem grew, simple measures of clinical physiology, biochemistry and behaviour were identified that operated at an epidemiological level as markers of cardiovascular risk. In the last two decades, this knowledge culminated in carefully organized randomised prospective controlled trials that have successfully demonstrated that intervention with drugs targeting these risk factors prevents cardiovascular events. The importance of these trials has been to show that pharmacological intervention, even at a late stage in the disease process, can influence natural history and clinical outcome. Furthermore, the identification of drugs with relatively specific effects has begun to clarify our understanding of the fundamental pathophysiological mechanisms promoting the progression of vascular disease

Another factor that has been instrumental in providing a deeper understanding has been the development of specialized techniques to examine the functions and control of the cardiovascular system. These have helped to define more clearly the changes that occur with age, the deleterious effects of classical risk factors and the response to established disease. Importantly, they have also allowed us to observe the impact of drugs on cardiovascular function. These techniques are also yielding new surrogate markers for cardiovascular disease in evolution. This is important for two reasons. In a world of limited resources there is clearly a need for more accurate definition of the 'at risk' population so that costly medicines can be targeted appropriately for greatest cost-effectiveness. Moreover, earlier intervention in the disease process can be expected to yield the greatest benefits in prevention of disease events. The same techniques have been particularly helpful in offering insights into the mechanism of drug action and identifying new targets for intervention. The need to gather accurate measurements has assumed further importance in the new era of molecular genetics. It is clear that to utilize the increasingly availability of genetic information from patients with complex traits (e.g. hypertension) to greatest potential we must be able to characterize accurately cardiovascular phenotype.

We have been invited by the Editorial Board of the British Journal of Clinical Pharmacology to commission and edit a series of prestigious review articles covering the most important research methods for studying the impact of drugs on cardiovascular function. The series will include manuscripts covering a variety of methods of measuring the function of the heart, the arterial system, the capillary bed, the venous system, and the autonomic nervous system. The articles have all been written by acknowledged authorities in these fields who are currently active in clinical research. In each case, we have asked the authors to provide a clear description of the current state of the art. Specifically, they were asked to provide enough depth and detail so that a competent clinical researcher with no previous experience of the method but a desire to set it up locally would find the justification to do so persuasive and the technical description comprehensive enough to follow. We hope you will find that these broad aims have been met.

\section{Simon Maxwell \& David Webb}

Clinical Pharmacology Unit, The University of Edinburgh, Western General Hospital, Porterfield Road, Edinburgh EH4 2LH 